\title{
Calibration of incremental temperature fluctuations at high temperatures
}

\author{
Marija Strojnik and Gonzalo Paez \\ Centro de Investigaciones en Óptica, Apdo. Postal 1-948, 37000 León, Gto., México, \\ mstrojnik@aol.com
}

\begin{abstract}
Both absolute and differential temperature measurements were simultaneously performed as a function of time for a pixel on a high-temperature, multi-spectral, spatially and temporally varying infrared target simulator. A scanning laser beam was used to maintain a pixel at an on-the-average constant temperature of $520 \mathrm{~K}$. The laser refresh rate of up to $1 \mathrm{kHz}$ resulted in small-amplitude temperature fluctuations with the peak-to-peak amplitude of less than $1 \mathrm{~K}$. The experimental setup to measure accurately the differential and the absolute temperature as a function of time is described.
\end{abstract}

\section{Introduction}

A prototype, high-temperature, spatially and temporally resolved infrared target simulator than can achieve heating rates on the order of $100 \mathrm{~K} / \mathrm{s}$ and can maintain high temperature gradients of $200 \mathrm{~K} / \mathrm{mm}$ was demonstrated. [1-3] The target consists of a low thermal diffusion material, such as glassy carbon. [4-6] A scanning high energy-density laser beam is used to create the required temperature distribution. [7,8] The scanning laser beam remains on each target pixel for a very short time, heating it very rapidly. When the laser beam is removed from the pixel, the pixel cools for an appreciably linger period of time. The pixel temperature, while constant on the average, undergoes short, small-amplitude fluctuations.

An infrared target simulator represents an object than may undergo smoothly varying temperature changes caused by external environment, including heating and cooling mechanisms, the simulator, when finished, may be used to test advanced trackers with anticipated temperature discrimination capabilities on the order of $1 \mathrm{~K}$ or less. [913] The high sensitivity of these trackers is achieved by using advanced detector and sophisticated pattern recognition algorithms that are still unknown and require development. [14,15].

Numerical modelling of heating with a scanning laser beam has shown that small temperature fluctuations are superimposed on top of the average temperature distribution. [16,17] Temperature variations of this kind may lead tracker to erroneous conclusions, since its decision-making algorithms rely on the tracker's rapid frame rate. Proper functioning of the simulator requires that it produce no artefacts. The simulator was tested to characterize the temporal profile of the pixel as a function of scanning parameters: the average pixel temperature, the duty cycle, and the scanning frequency. This paper describes the experimental setup to measure the precise temperature of a pixel at the laser scanning rate of up to $1 \mathrm{kHz}$, with the expected peak-to-peak temperature amplitude of less than $1 \mathrm{~K}$ at $520 \mathrm{~K}$. 


\section{Experimental setup}

Temperature distribution over a target surface can be effectively measured as a function of time, using an infrared camera. This may be appropriate when the temperature distribution varies slowly compared to the infrared camera scanning rate. For the case when a target pixel is exposed to a scanning high-energy density laser beam at a $1 \mathrm{kHz}$ rate, a higher temporal resolution than that provided by the infrared camera is needed in order to assess the exact temperature profile.

A radiometer (calibrated for the required temperature range, speed of response, and field of view) is required for the measurement of temperature that rapidly varies with time.

Three setups are needed to measure the temporal profile of the pixel temperature: the calibrations setup, the temperature measurement setup, and the temperature fluctuation measurement setup. Figure 1 shows the calibration setup. The imaging optics images opening of the blackbody simulator onto the detector. A radiation chopper and a long wavelength cut-off filter are used in front of the detector. The filter is necessary during the measurement setup to eliminate the laser radiation which reflects of the target and other surfaces. The output of the radiation chopper is used to trigger the time base on the oscilloscope.

A nearly perfect square wave ensures clearly defined regions for the radiation and the reference inputs. The oscilloscope measures the voltage difference corresponding to the difference in temperature between the cold blades of the chopper and the blackbody radiation source. Thus, the relationship between the output voltage of the detector and the temperature of the blackbody is established. Figure 2 shows the experimentally measured voltage-temperature calibration curve.

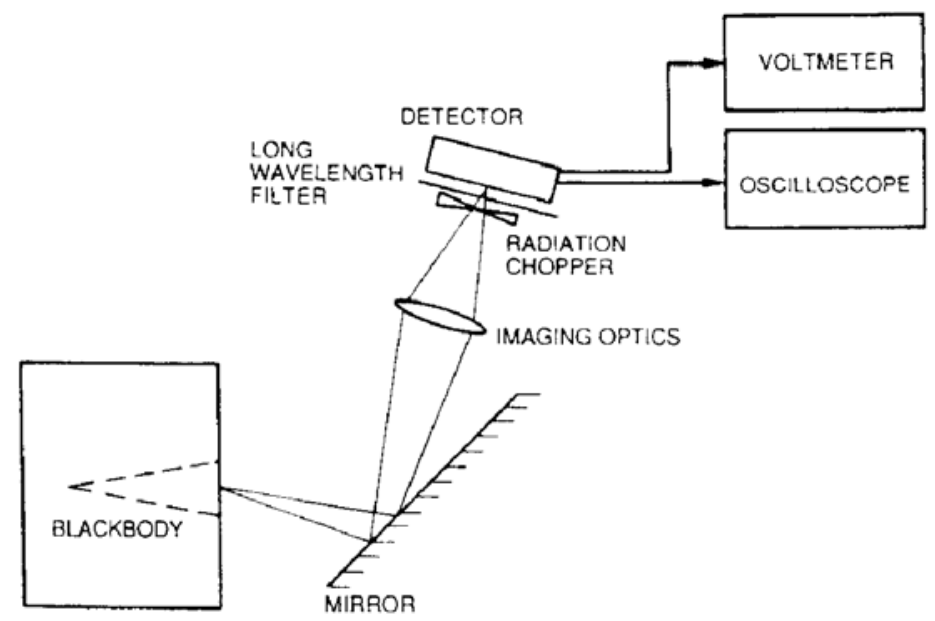

Fig. 1. Experimental setup for detector calibration when measuring miniscule temperature ripple. 


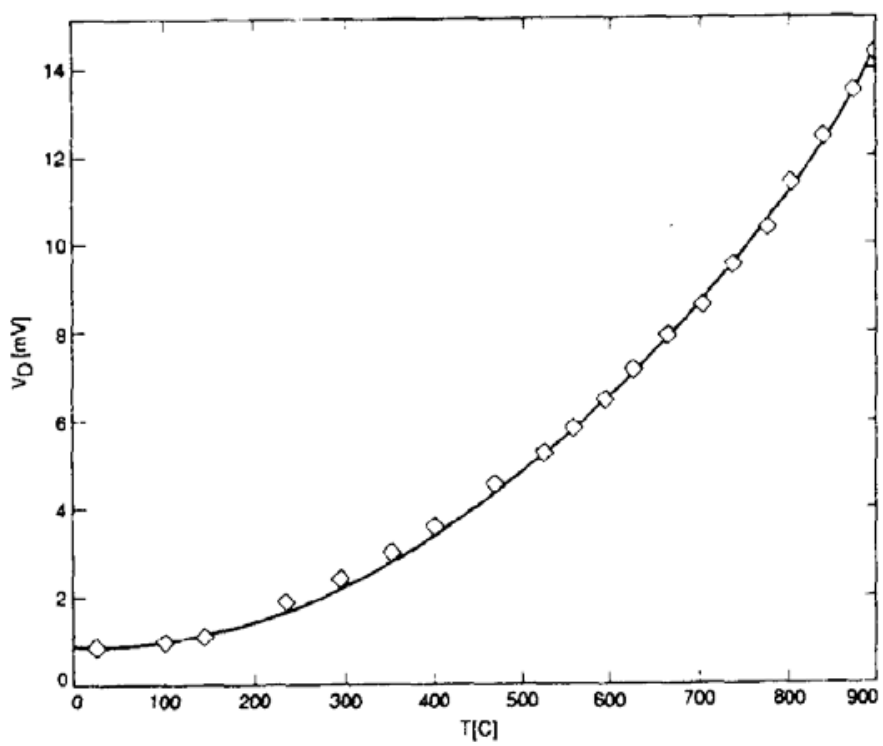

Fig. 2. Detector output voltage is a quadratic function of reference temperature.

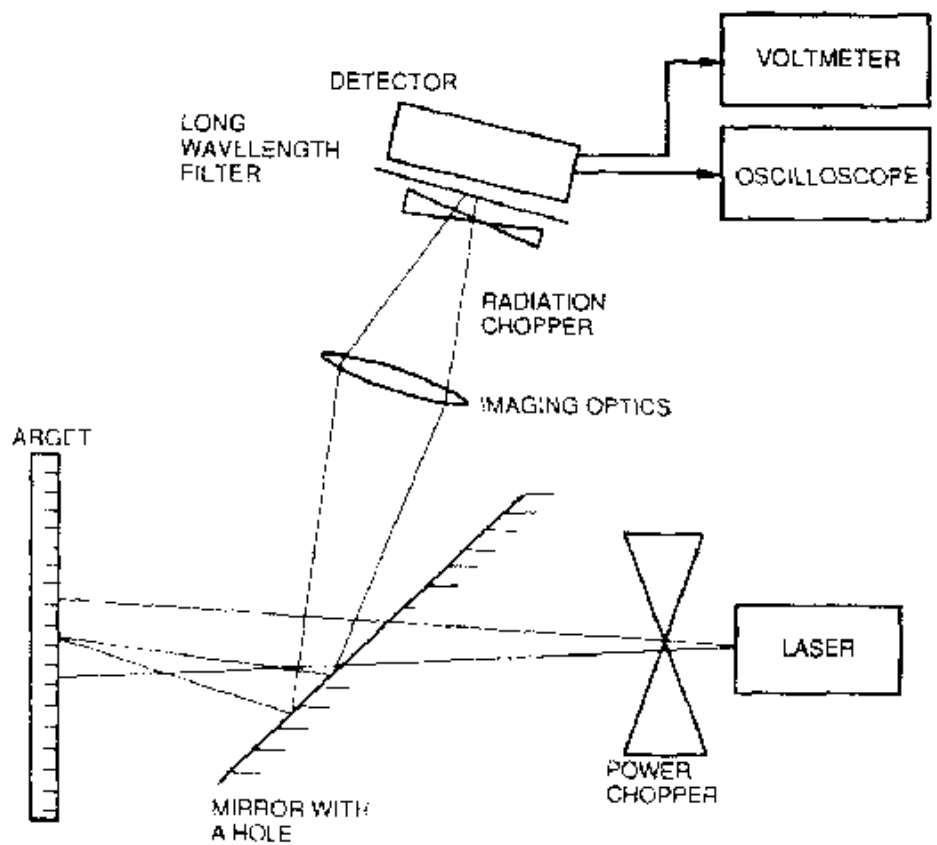

Fig. 3. Experimental setup to measure temperature of the target, irradiated by a high-power pulsed beam in near IR. 
The second step in this procedure is the temperature measurement stage. In this part of the experiment, the blackbody is replaced with the target, as shown in Fig. 3 . The combination of the laser and the power chopper form the source of power. The components are arranged to make the optimum use of the laser power and to maximize the signal: the irradiated pixel just fills the field of view of the detector at the target.

A $100 \mathrm{~W}$ nominal power Nd YAG laser at $1.068 \mu \mathrm{m}$ is used to maintain the pixel temperature $200 \mathrm{~K}$ above the background temperature. A cooled, single element $\mathrm{HgCdTe}$ detector sensitive in $8 \mu \mathrm{m}$ wavelength interval is used to measure the temperature. In this setup, the scanning laser beam is simulated with a power chopper. The laser beam is aligned with the irradiated target element.

The aperture scans across the laser beam to simulate scanning. The scanning aperture is shown in Figure 4. It is possible to shown analytically that equal surface incidence is obtained when the laser beam scans over the target surface, or in this case when a circular aperture scans over the laser beam.

Several holes are drilled along the circumference of a wheel. The number of holes and the rotational speed of the wheel determine the frequency of irradiation. The angle subtended by the holes and their radius determine the duty cycle of the

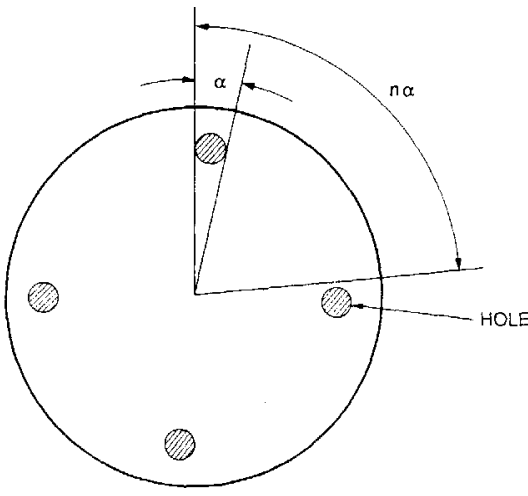

Fig. 4. Power chopper with duty cycle $1 / \mathrm{n}$. irradiated beam

The setup used to measure the temperature fluctuations is similar to the temperature measurement setup (see Figure 3), except that the voltmeter is replaced with a lockin amplifier. After the laser power has been set to generate the required hot spot temperature, the output of the detector is used as the input to the lock-in amplifier or the oscilloscope. The output from the power chopper is used as the reference to the second lock-in amplifier, or as the source for the external trigger on the oscilloscope.

\section{Analysis}

The measured temperature ripple is too small to be observed on the oscilloscope due to the noise level of about $1 \mathrm{~K}$, when the pixel temperature is $220 \mathrm{~K}$ above the background. Therefore, a lock-in amplifier is used to measure the amplitude of the temperature fluctuations indirectly. The relationship between the RMS voltage of the harmonic measured with the lock-in amplifier and the temperature peak-to-peak amplitude is developed in this section.

The amplitude of the temperature fluctuations increases with pixel temperature; therefore, the exact trace of the temperature-vs-time profile can be observed at 


\section{http://dx.doi.org/10.21611/qirt.2006.079}

higher pixel temperatures. When the irradiated pixel is about $500 \mathrm{~K}$ above the background temperature (as compared with $220 \mathrm{~K}$ ) the waveform of the temperature fluctuations may be observed on the oscilloscope despite the presence of a $1 \mathrm{~K} \mathrm{RMS}$ noise. A lock-in amplifier is used simultaneously to measure the RMS voltage of the fundamental wave of the waveform. The relationship between the peak-to-peak voltage due to the temperature fluctuation and the RMS voltage, measured with the lock-in-amplifier, is obtained.

The differential relationship between the voltage and temperature is obtained in the calibration step (see Figure 2). The relationship between the change in temperature and change in voltage is obtained with the help of Figure 5 . The temperature $T_{0}$ produces a voltage increase of $V_{0}$. In the neighbourhood of $V_{0}$, the voltage may be expanded in a Taylor series: After some manipulation, we obtain the desired results.

$$
\Delta \mathrm{T}_{\mathrm{P}-\mathrm{P}}(\mathrm{T})=\alpha(\mathrm{dT} / \mathrm{dV}) \Delta \mathrm{V}_{\mathrm{RMS}}(\mathrm{T})
$$

The peak-to-peak voltage (P-P) and the RMS voltage measured with the lock-in amplifier are related with the proportionality factor $\alpha$. Derivative of voltage with respect to temperature may be found by numerically differentiating the data in Fig. 2 .

\section{Results}

The peak-to-peak amplitude of the temperature fluctuations was measured as a function of the scanning frequency for a $10 \%$ and a $20 \%$ duty cycle, for the average pixel temperature of $250 \mathrm{C}$. Figure 6 shows that the magnitude of the temperature fluctuations decreases with increasing scanning frequency. The rate of the decrease in the magnitude of the fluctuations is larger for a longer duty cycle. There appears to be a cross-over point for the magnitude of the temperature fluctuations at about 70 $\mathrm{Hz}$.

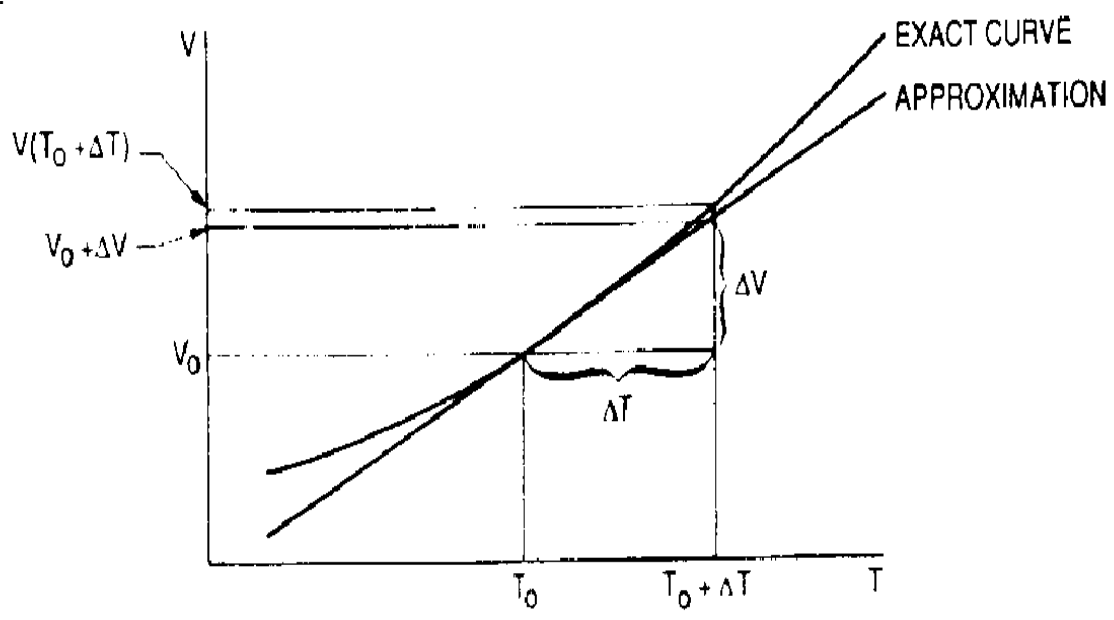

Fig. 5. Typical relationship between the voltage output of a detector and the reference temperature. 


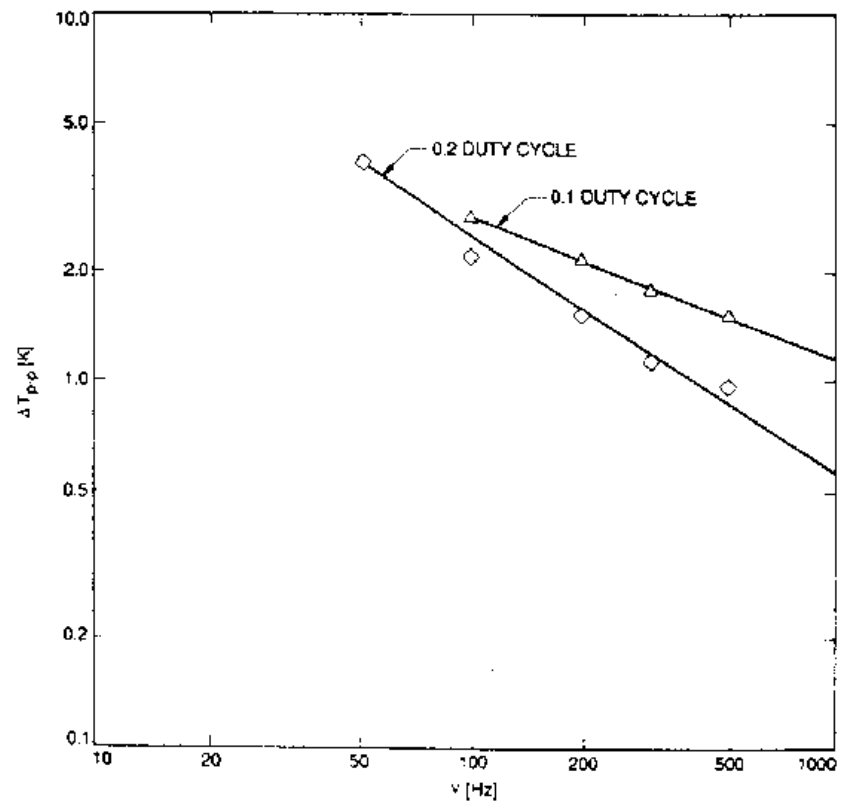

Fig. 6. Temperature peak-to-peak fluctuations as functions of pixel temperature.

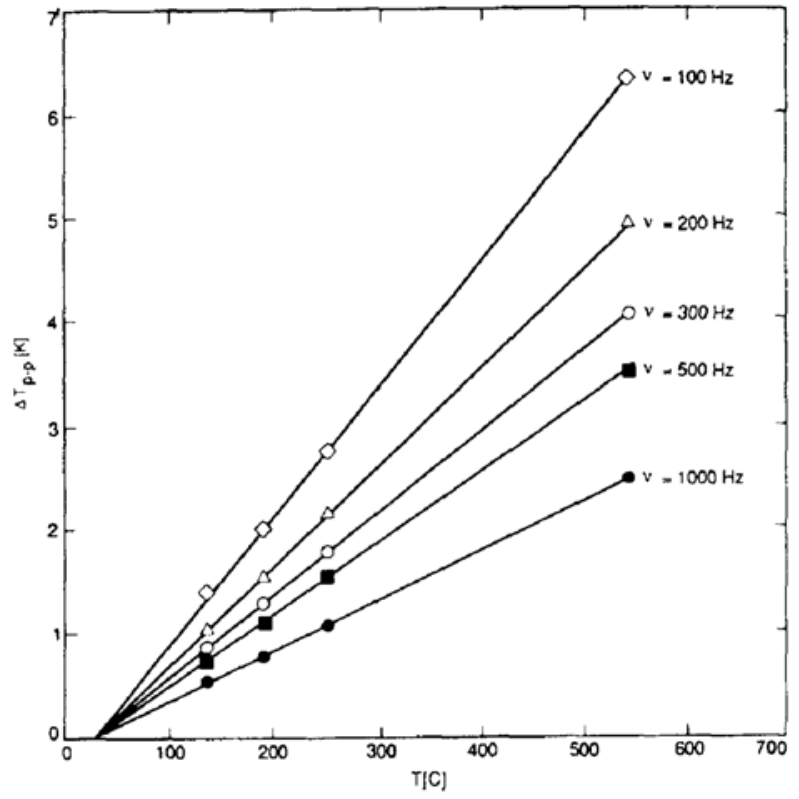

Fig. 7. Temperature peak-to-peak fluctuations as functions of pixel temperature. 
Figure 7 presents the peak-to-peak amplitude of the temperature fluctuations as a function of the pixel temperature. The amplitude of the temperature fluctuations was determined using the method presented here for the $\Delta T_{\mathrm{P}-\mathrm{P}}$ values of less than $2 \mathrm{~K}$ in the presence of $1 \mathrm{~K}_{\mathrm{RMS}}$ noise. Measurements at higher temperatures were performed for the purposes of calibrating the factor $\alpha$.

The peak-to-peak amplitude of the temperature fluctuations was also measured as a function of the duty cycle, for the average pixel temperature of $250 \mathrm{C}$. The magnitude of the peak-to-peak temperature fluctuations decreases by a factor of two when the refresh rate is doubled. Thus, the refresh rate appears to be the most important parameter in decreasing the peak-to-peak temperature fluctuations.

\section{Conclusions}

Accurate determinations of the temperature fluctuations of less than $1 \mathrm{~K}$ peak-topeak amplitude were obtained using the experimental method described in this paper. The peak-to-peak voltage and the RMS voltage of the first harmonic measured with the lock-in amplifier are related upon visualization of the temperature waveform at high-temperatures. The amplitude increases linearly with temperature of the surface and decreases logarithmically with frequency.

\section{References}

1. M. D. Kelly, L. D. Abney, "Fast times at high temperatures," Thermosense VII, Proc. SPIE 520 (1984).

2. M. S. Scholl, "Thermal Considerations in the Design of a Dynamic IR source," Appl. Opt., 21 (4), 660-667 (1982).

3. J. M. Milne, W. N. Raynolds, "Non-destructive evaluation of composites and other materials with thermal pulse video thermography," Thermosense VII, Proc. SPIE 520 (1984).

4. M. S. Scholl, W. L. Wolfe, "An infrared target design - fabrication considerations," Appl. Opt., 20 (12), 2143-2152 (1981).

5. M. S. Scholl, "Spatial and temporal effects due to target Irradiation: A study," Appl. Opt., 21 (9), 1615-1620 (1982).

6. M. S. Scholl, "Time- and Positions-Varying Infrared Scene Simulation," in Infrared Technology XIII, Proc. SPIE 819 (1987).

7. M. S. Scholl, "Target temperature distribution generated and maintained by a Scanning Laser Beam," Appl. Opt., 21 (12), 2146-2152 (1982).

8. M. S. Scholl, "Measurement of small temperature fluctuations at high average temperature,"'Infrared Technology XIV, Proc. SPIE 972, 409-415 (1988). 
9. J. Hopper, S. Bauman, N. Faust, R. Smith, M. Weathersby, "A digital infrared imagery data collection and analysis system," Thermal Imaging, Proc. SPIE 636 (1986).

10. C. L. Wyatt, "Radiometric linearity calibration,"-Infrared Technology XI, Proc. SPIE 572 (1985).

11. R. Fourier, R. A. Buckwald, D. Cabib, E. Sapir, "Microprocessor-based radiation sources and radiometers for testing thermal imaging systems," Thermosense VII, Proc. SPIE 520 (1984).

12. J. Hopper, S. Bauman, N. Faust, R. Smith, M. Weathersby, "A Digital Infrared Imagery Data Collection and Analysis System," in Thermal Imaging,_Proc. SPIE 636 (1986).

13. C. L. Wyatt, "Radiometric Linearity Calibration," in Infrared Technology XI, Proc. SPIE 572 (1985).

14. J. Merchant, "Interframe image processing," in Thermal Imaging,_Proc. SPIE 636 (1986).

15. M. S. Scholl, "Errors in radiance simulation and scene discrimination," Appl. Opt., 21 (10), 1839-1843 (1982).

16. M. S. Scholl, Simulation of spectral radiance of a dynamic IR source, Ph. D. Dissertation, University of Arizona, Tucson, Arizona (1979).

17. G. Paez and M. Strojnik, "Measurement and calibration of temporal and spatial temperature differences of $100 \mathrm{~K}$," elsewhere in these Proceedings. 